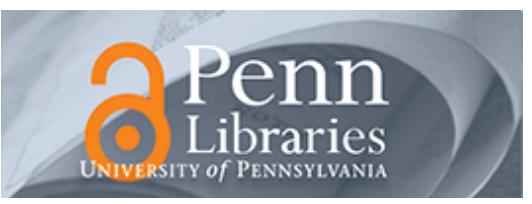

University of Pennsylvania

ScholarlyCommons

\title{
Statistically-Constrained High-Dimensional Warping Using Wavelet-Based Priors
}

\author{
Zhong Zue \\ zhong.xue@uphs.upenn.edu \\ Dinggang Shen \\ University of Pennsylvania, Dinggang.Shen@uphs.upenn.edu \\ Christos Davatzikos \\ University of Pennsylvania, christos@rad.upenn.edu
}

Follow this and additional works at: https://repository.upenn.edu/be_papers

Part of the Biomedical Engineering and Bioengineering Commons

\section{Recommended Citation}

Zue, Z., Shen, D., \& Davatzikos, C. (2006). Statistically-Constrained High-Dimensional Warping Using Wavelet-Based Priors. Retrieved from https://repository.upenn.edu/be_papers/159

Suggested Citation:

Xue, Z., D. Shen, and C. Davatzikos. (2006). "Statistically-Constrained High-Dimensional Warping Using WaveletBased Priors." Proceedings of the 2006 Conference on Computer Vision and Patern Recognition Workshop. June 17-22, 2006.

C2006 IEEE. Personal use of this material is permitted. However, permission to reprint/republish this material for advertising or promotional purposes or for creating new collective works for resale or redistribution to servers or lists, or to reuse any copyrighted component of this work in other works must be obtained from the IEEE.

This paper is posted at ScholarlyCommons. https://repository.upenn.edu/be_papers/159

For more information, please contact repository@pobox.upenn.edu. 


\title{
Statistically-Constrained High-Dimensional Warping Using Wavelet-Based Priors
}

\author{
Abstract \\ In this paper, a Statistical Model of Deformation (SMD) that captures the statistical prior distribution of \\ high-dimensional deformations more accurately and effectively than conventional PCA-based statistical \\ shape models is used to regularize deformable registration. SMD utilizes a wavelet-based representation \\ of statistical variation of a deformation field and its Jacobian, and it is able to capture both global and fine \\ shape detail without overconstraining the deformation process. This approach is shown to produce more \\ accurate and robust registration results in MR brain images, relative to the registration methods that use \\ Laplacian-based smoothness constraints of deformation fields. In experiments, we evaluate the SMD- \\ constrained registration by comparing the performance of registration with and without SMD in a specific \\ deformable registration framework. The proposed method can potentially incorporate various registration \\ algorithms to improve their robustness and stability using statistically-based regularization.

\section{Disciplines} \\ Biomedical Engineering and Bioengineering | Engineering

\section{Comments} \\ Suggested Citation: \\ Xue, Z., D. Shen, and C. Davatzikos. (2006). "Statistically-Constrained High-Dimensional Warping Using \\ Wavelet-Based Priors." Proceedings of the 2006 Conference on Computer Vision and Patern Recognition \\ Workshop. June 17-22, 2006. \\ C2006 IEEE. Personal use of this material is permitted. However, permission to reprint/republish this \\ material for advertising or promotional purposes or for creating new collective works for resale or \\ redistribution to servers or lists, or to reuse any copyrighted component of this work in other works must \\ be obtained from the IEEE.
}




\title{
Statistically-Constrained High-Dimensional Warping Using Wavelet-Based Priors
}

\author{
Zhong Xue, Dinggang Shen, and Christos Davatzikos \\ Section of Biomedical Image Analysis (SBIA), Department of Radiology \\ University of Pennsylvania, Philadelphia, Pennsylvania \\ zhong.xue@uphs.upenn.edu
}

\begin{abstract}
In this paper, a Statistical Model of Deformation (SMD) that captures the statistical prior distribution of highdimensional deformations more accurately and effectively than conventional PCA-based statistical shape models is used to regularize deformable registration. SMD utilizes a wavelet-based representation of statistical variation of a deformation field and its Jacobian, and it is able to capture both global and fine shape detail without overconstraining the deformation process. This approach is shown to produce more accurate and robust registration results in $M R$ brain images, relative to the registration methods that use Laplacian-based smoothness constraints of deformation fields. In experiments, we evaluate the SMD-constrained registration by comparing the performance of registration with and without SMD in a specific deformable registration framework. The proposed method can potentially incorporate various registration algorithms to improve their robustness and stability using statistically-based regularization.
\end{abstract}

\section{Introduction}

Many registration methods have been proposed for finding the deformation field between two 3D images by maximizing the image-similarity measure and, at the same time, properly constraining/regularizing the deformation field [7, $9,15,16,17]$. In these methods, a variety of smoothness constraints are used, such as Laplacian-based regularization, or physically-based constraints, e.g. elasticity and viscoelasticity. Statistical models have also been utilized to regularize the registration procedure to improve the registration performance [18]. Compared to the conventional methods, statistically-constrained deformable registration can achieve more robust performance, because the statistical regularization constraints reflect the relatively complex nature of the respective deformation fields.

Estimating the statistics or the probability density func- tion (pdf) of 3D or 4D deformation fields from a limited number of training samples is a very challenging task. One of the most popular methods has been the application of Principal Component Analysis (PCA) [3, 4, 12], in order to estimate a number of principal components that are frequently called principal modes of shape variation. However, this approach often fails when applied to 3D dense deformation fields, due to under-training in practical settings. For example, accurately estimating a dense 3D deformation field of the entire brain could require tens of thousands of training deformations, if not more. Accordingly, in the 1990's, methods based on scale-space decompositions were investigated [11], allowing us to analyze information at different scales. In this paper, we use the statistical model of [21], referred to as Statistical Model of Deformation (SMD), to capture the statistics of deformations. SMD builds upon the methods described in $[2,6,13]$, which use Wavelet Packet Transform (WPT) coupled with PCA in each wavelet band, in order to more accurately estimate pdfs of high-dimensional deformation fields, when only a relatively small number of training samples are available (e.g. tens or in the order of 100).

The basic premise in SMD is that a number of deformation fields defined in the standard template image domain are available to be used for training. Defining such training samples is not an easy task, and its full treatment is beyond the scope of this paper. For the purposes of demonstrating and testing our methodology, we used a number of deformations obtained by using the high-dimensional deformable registration method, called HAMMER [16]. This inevitably biases the generated deformations toward the family of deformations that can be generated by this particular warping algorithm. This is in agreement with our goal here, which is to construct effective statistical priors for constraining this specific deformable registration mechanism. However, this statistically-based regularization approach can be applied in conjunction with any registration algorithm. We show that the performance of the registration algorithm [16] is further improved after constraining it by the statistical model. 
Moreover, training samples can ultimately be generated from first extensively labeling and landmarking a number of training images [1], and then applying a high-dimensional warping algorithm to these images, where these labels and landmarks act as the constraints of the deformation fields. Such adequately constrained warping algorithms are likely to generate deformations that are close enough to a gold standard, and therefore appropriate for training.

After obtaining SMD, we use it as prior knowledge to iteratively constrain the deformable registration. In experiments, we evaluate the SMD-constrained registration by comparing the performance of registration with and without SMD in a specific deformable registration framework, by registering simulated and real MR brain images.

\section{Method}

We first introduce the Statistical Model of Deformation (SMD) [21] for capturing the statistics of deformations and then describe the SMD-constrained deformable registration.

\subsection{Statistical model of deformation}

Denoting $\mathbf{f}(\mathbf{x}), \mathbf{x} \in \Omega_{t}$, as the deformation field defined over the template image domain $\Omega_{t}$, the goal of SMD is to estimate the pdf of $\mathbf{f}$, i.e. $p(\mathbf{f})$, from a relatively small number of training samples. In order to capture finer and more localized variations of $\mathbf{f}$, SMD follows and extends the framework proposed in [6], which is referred to as the Wavelet-based PCA (W-PCA) model. The W-PCA model decomposes $\mathbf{f}$ using the Wavelet-Packet Transform (WPT) and subsequently captures within-scale statistics using PCA in each wavelet band. The fundamental assumption in W-PCA is that the wavelet-based rotation renders the covariance matrix of deformation $\mathbf{f}$ close to block-diagonal, thereby enabling a more accurate estimation of the statistical distribution in each block (wavelet band) from a limited set of examples, compared to the usual sample covariance estimation, due to both of lower dimensionality and relatively strong correlations among variables.

In theory, if the W-PCA model captures the statistics of deformation $\mathbf{f}$ accurately, we can just use it as the statistical model. In practice, however, the assumption that the covariance matrix of $\mathbf{f}$ is block-diagonal in the wavelet-packet basis does not hold exactly. Although it is well-known that for broad classes of signals, correlations across scales diminish rapidly, they are nonetheless non-negligible for adjacent scales. In order to alleviate this problem, we observe that additional constraints imposed on the deformation fields can be used to define subspaces in which the deformation must belong. Therefore, SMD requires that a valid deformation field belongs to the intersection of three subspaces, each of which satisfies some constraints on the deformation. The W-PCA model applied to the deformation field speci- fies the first subspace.

In order to describe the second subspace, we use the WPCA model of the Jacobian determinants of the deformation fields, since they reflect local volumes of anatomical structures, which are important from the perspective of spatial distribution of the amount of brain tissue. In fact, anatomical structures from different subjects can differ quite dramatically in shape, but not always in volume. Thus, it would be reasonable to assume that, although the cortical folding patterns can vary wildly across individuals, the need of different cortical structures to occupy certain tissue volume renders the Jacobian determinant, which is directly related to tissue volume, relatively small variable across individuals, and therefore easier to statistically characterize Jacobian determinants from a limited number of samples. Therefore, we require that a valid deformation field be subject to the constraints defined by the W-PCA models of both deformations and Jacobian determinants. Note that converting a constraint on the Jacobian determinant to a constraint on the deformation field is a challenging task with no unique solution. Herein we use [10], which utilizes an iterative projection scheme that minimally, according to some distance criteria, to modify a given displacement field so that it satisfies certain conditions on the Jacobian determinant. This algorithm is used to realize the projection of a given displacement field to the subspace of "valid Jacobians".

To define the third subspace, we note that if deformations are synthesized directly using the W-PCA model, some unrealistic discontinuities emanating from the assumption of statistical independence across wavelet bands may occur. Therefore, a nested Markov Random Field (MRF) regularization scheme is applied to eliminate such potential discontinuities.

In Summary, SMD combines the W-PCA models of deformation fields and their Jacobian determinants, as well as the MRF regularization, and requires that a valid deformation field locates inside the intersection of the three subspaces defined by these models. Given an input deformation field, we can iteratively project it onto each of the three subspaces, and finally generate the SMD-regularized deformation field according to the priors. This SMD regularization algorithm is summarized as follows,

Step 1. Project the deformation field onto the W-PCA model of valid deformation fields;

Step 2. Project the Jacobian of the deformation field onto the W-PCA model of valid Jacobian determinants;

Step 3. Modify the deformation field so that its Jacobian determinants match the target Jacobians generated in Step 2 , and at the same time, subject to certain smoothness constraints on the deformation field (refer to [10] for details);

Step 4. Apply the nested MRF regularization to impose spatial smoothness on the deformation at all scales;

Step 5. Go to step 1 and iterate until convergence, i.e. 


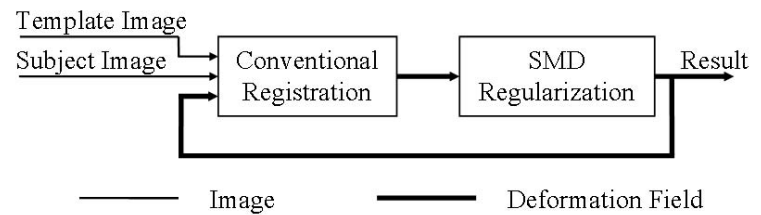

Figure 1. The structure of the SMD-constrained deformable registration, wherein the SMD regularization is incorporated with a conventional registration algorithm in an iterative procedure.

until the MRF-regularized deformation field belongs to the subspaces of valid Jacobians and deformations.

The ability of SMD to capture the statistical variation of deformation fields resulting from brain image warping was tested by simulating various of new deformation fields and respective brain images [21].

\subsection{SMD-constrained deformable registration}

After estimating the statistical model of deformation fields, we can use this prior knowledge to constrain the deformation field during image registration. The structure of the SMD-constrained deformable registration is shown in Fig.1. The SMD-constrained registration iteratively regularize the deformation field generated by a conventional registration algorithm using the SMD regularization algorithm described in Section 2.1, and it improves the robustness and stability of the registration results since prior statistical information about the variability of deformation fields has been embedded into the registration procedure.

Particularly, the SMD-constrained deformable registration is achieved by estimating a deformation field $\mathbf{f}(\mathbf{x})$, which defines the voxel-wise displacements from the template image $I_{t}$ onto the subject image $I_{s}$, by minimizing the following energy function,

$$
\begin{aligned}
E= & \lambda_{S M D} E_{S M D}(\mathbf{f}) \\
& +\lambda_{S} E_{S}(\mathbf{f})+\lambda_{S I M} E_{S I M}\left(I_{t}, I_{s} \mid \mathbf{f}\right),
\end{aligned}
$$

where the energy term $E_{S M D}$ reflects the statistical constraint of the deformation field, i.e. SMD regularization, the energy term $E_{S}(\mathbf{f})$ is a regular smoothness constraint such as Laplacian-based regularization of the deformation field, and $E_{S I M}$ is the energy term reflecting the image-similarity between the template image $I_{t}$ and the subject image $I_{s}$ under current deformation field f. Image intensity-based similarity, mutual information [19], and attribute vectorbased image similarity measures [16] could be used for $E_{S I M}$. Compared to the conventional registration methods that only utilize the smoothness constraint term and the image-similarity term, the SMD-constrained registration uses a new statistical regularization energy term $E_{S M D}$ so that the resultant deformation field conforms to the prior knowledge defined by SMD.
Notice that the energy function of Eq.(1) consists of two parts. The first part includes the statistical regularization using SMD, and the second part represents the energy function defined by a conventional registration algorithm, including the smoothness constraint and the image-similarity measure. Therefore, the SMD-constrained registration can be achieved by iteratively regularizing the deformation field estimated by the registration algorithm using SMD. In the first iteration, we use the registration algorithm to estimate a new deformation field between the template image and the subject image and then regularize the deformation field using the SMD regularization algorithm described in Section 2.1 ; in the subsequent iterations, the regularized deformation field is used as the input (or initial deformation) of the registration algorithm in order to iteratively refine the registration result. This process terminates until the difference between the resultant deformation fields of two subsequent iterations drops below a certain threshold.

We have used this approach in conjunction with the HAMMER registration algorithm. However, any other conventional registration algorithm could be used instead. Our perspective of this approach is that we treat the family of all possible deformations generated by the registration algorithm as the fourth subspace, and iteratively project the deformation field onto each of the four subspaces until it locates in the intersection of all of the four subspaces. One could even use multiple registration algorithms in this framework.

\section{Results}

The performance of the SMD-constrained registration framework is evaluated on both simulated and real MR brain images, by comparing HAMMER [16], which is available from our website https://www.rad.upenn.edu/sbia/rsoftware.html, and its SMD-constrained version, SMD+HAMMER. The SMD is trained using 158 inter-individual deformation fields that register a template image onto 158 BLSA data, respectively [14]. In the first experiment, we examine the ability of the two registration methods to detect morphological differences (atrophy) simulated in two groups of brain scans, by examining quantities extracted from the deformation fields linking a template with each image in these two groups, i.e. using a computational anatomy approach [5]. Moreover, other measurements, such as smoothness of deformation field, are also compared. Two additional experiments are performed on real data. First, the registration accuracy of each registration method is measured by a number of expert-defined anatomical landmarks. Second, serial images of the same subjects are aligned onto the template space, and the temporal consistency/smoothness of the registration results is measured quantitatively and also visually for each registration method. The premise in the last 

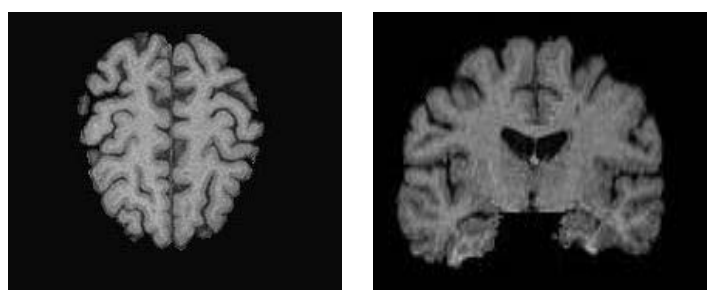

(a) Original image
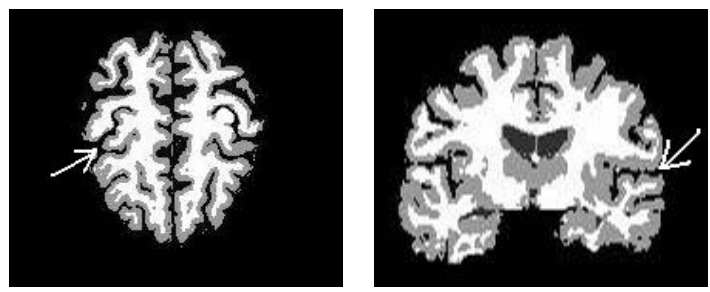

(b) Segmented image
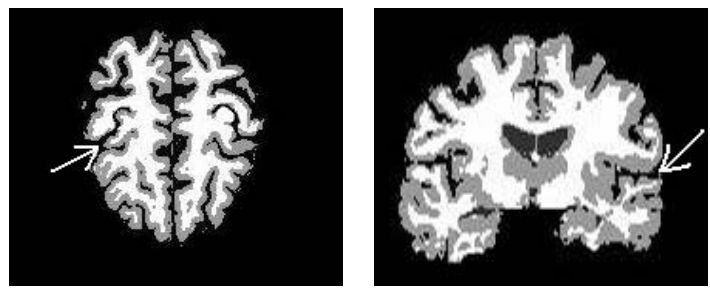

(c) Simulated atrophy

Figure 2. The original MR brain image, the segmented image and the simulated image with local atrophy on precentral gyrus and superior temporal gyrus (indicated by arrows).

experiment is that the transformations that yield temporally consistent results are likely to be more accurate, since the input images are acquired from the same individual in consecutive years, and they differ very little from year to year.

\subsection{Registration of simulated atrophy images}

We use both HAMMER and SMD+HAMMER to register the MR brain images of different subjects with and without simulated atrophy. $10 \mathrm{~T} 1$-weighted MR brain images of 10 different subjects are used, referred to as the group of original brains. For each original image, we simulate the atrophy on both precentral gyrus and superior temporal gyrus [5]. Therefore, we obtain 10 simulated atrophy images, referred to as the group of simulated brains. Fig.2 shows an original MR brain image and its simulated atrophy image.

All the 20 images, 10 in the original group and 10 in the simulated group, are then registered onto the template image by using HAMMER and SMD+HAMMER, respectively. To evaluate the performance of the registration results, we perform the following quantitative comparisons.

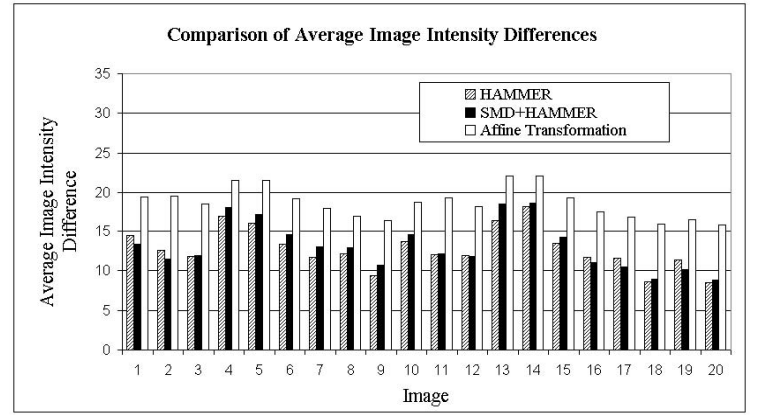

Figure 3. Comparison of average image intensity difference between the template image and each registered subject image.

\subsubsection{Image intensity difference and smoothness of de- formation field}

The average image intensity difference and the smoothness of deformation field are used to evaluate the performance of the registration, since they give us an idea about the goodness of registration. In general, good registration results are reflected by small image intensity differences and smooth deformation fields. In this paper, the average image intensity difference between the registered subject image $I_{s}$ and the template image $I_{t}$ is calculated by,

$e\left(I_{t}, I_{s}, \mathbf{f}\right)=\frac{\iiint_{\Omega_{t}}\left[I_{t}(\mathbf{x})-I_{s}(\mathbf{f}(\mathbf{x}))\right]^{2} d x_{1} d x_{2} d x_{3}}{\iiint_{\Omega_{t}} d x_{1} d x_{2} d x_{3}}$,

where $x_{1}, x_{2}$, and $x_{3}$ are the elements of voxel $\mathbf{x} \in \Omega_{t}$. The smoothness of a deformation field is evaluated using the histogram of the Jacobian determinants and the histogram of the Laplacian magnitudes of that deformation field, respectively.

Fig. 3 shows the average image intensity difference between the template and each registered subject image. It can be seen that the average image intensity difference is similar for both HAMMER and SMD+HAMMER registration results, and there is no significant difference between the results of these two registration methods. That means the registration accuracy is not decreased by using SMD as additional constraints for deformation field, which would happen if SMD over constrained the deformation. For reference, the average image intensity difference of the poor affine registration is also shown. Notice that the average image intensity difference is still large even for the HAMMER and SMD+HAMMER algorithms, since the image intensities are not globally normalized between the template and each subject image, when calculating the average image intensity difference.

Fig.4 and Fig.5 compare the histograms of the Jacobian determinants and the histograms of the Laplacian magnitudes of a deformation field generated by HAMMER 


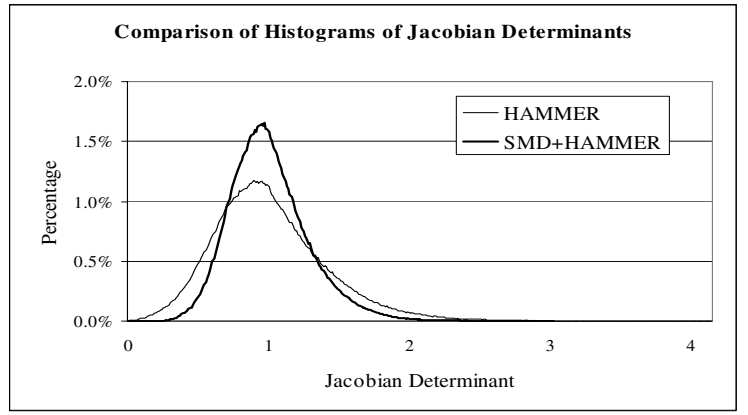

Figure 4. Comparison of Jacobian determinants.

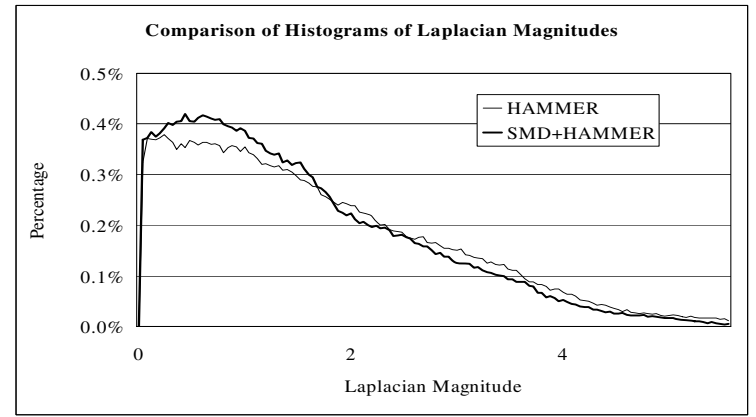

Figure 5. Comparison of Jacobian determinants.

and SMD+HAMMER, respectively. It can be seen that for SMD+HAMMER, the Jacobian determinants are more tightly distributed around one, and the Laplacian magnitudes are relatively small, compared to HAMMER.

The resultant deformation fields can also be visually observed in Fig.6. We can see that HAMMER and SMD+HAMMER generate similar deformation fields for the same template and subject image pair, but the SMD+HAMMER result is relatively smooth. Moreover, in some locations pointed by the arrows, the deformation field is clearly regularized by SMD+HAMMER, as compared with the HAMMER result.

These results indicate that by incorporating SMD into the registration procedure, we can obtain relatively smooth deformation fields, without decreasing registration accuracy. Further evaluation of registration accuracy is also performed by measuring the accuracy in aligning manual landmarks in real images as provided in Section 3.2.1.

\subsubsection{Ability to detect simulated atrophy}

We used a computational anatomy method to detect group differences between the brains with and without atrophy. For each brain image, we calculated the tissue density maps for different brain tissues in the template image space, i.e. the RAVENS maps $[5,8,14]$ of gray matter (GM), white
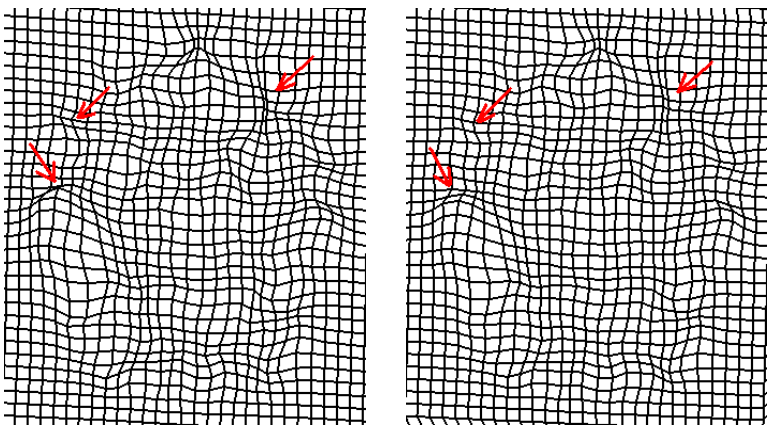

Figure 6. Examples of the deformation fields generated by HAMMER and SMD+HAMMER, respectively. Arrows indicate areas of local improvement of the deformation field.

matter (WM), and the cerebrospinal fluid inside the ventricle ( $\mathrm{VN})$, from the deformation field that registers this brain onto the template space. These tissue density maps are created in a mass-preserving way, and directly reflect the regional volumetric structure of the respective brains. For example, if an individual's ventricle is deformed into conformation with a template's ventricle, local expansion or contraction changes the local density of $\mathrm{VN}$, i.e. trying to match large subject ventricles to smaller template ventricles increases the local density. This is also true for GM and WM structures, as well as any subdivisions of them. Since the original information about volumes of brain structures and any arbitrary partitions of them is converted into tissue density maps in the template space, local differences or changes in volumes can be quantified by respective changes in these maps. Fig.7 shows the examples of the brain tissue density maps for GM, WM, and VN, calculated from HAMMER and SMD+HAMMER results, respectively. It can be seen that relatively smooth tissue density maps were obtained by SMD+HAMMER. By smoothness here, we mean that abrupt changes in tissue density, which adversely offset voxel-based statistical analysis of these tissue density maps, are reduced, allowing us to better detect the atrophy.

In order to test for group differences, we performed a paired t-test on the brain density maps of the two groups (each group includes 10 brain density maps calculated from 10 respective deformation fields), using the Statistical Parametric Mapping (SPM) software package. A smaller $\mathrm{p}$-value or a larger t-value of a paired t-test will indicate better separation ability and detection power. Table 1 shows the statistical measures for the two clusters detected in the locations of the superior temporal gyrus and the precentral gyrus, respectively. It can be seen that smaller p-values (both of $P_{F W E-c o r r}$ and $P_{F D R-c o r r}$ ) and larger t-values are obtained for SMD+HAMMER. Therefore, SMD+HAMMER in these experiments was much more powerful in detecting group differences. 

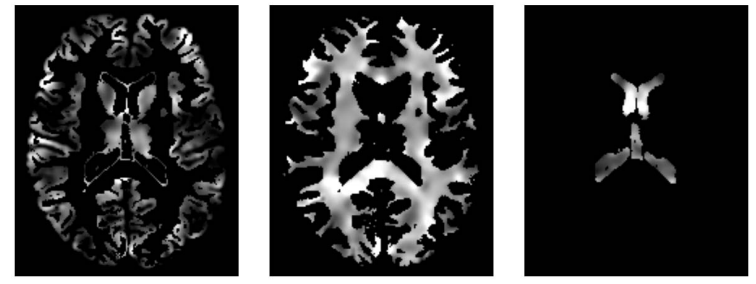

(a) HAMMER
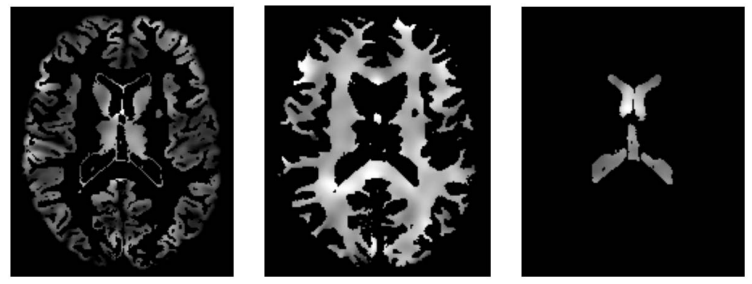

(b) SMD+HAMMER

Figure 7. Examples of the tissue density maps for GM, WM and $\mathrm{VN}$, calculated from the deformation fields generated by HAMMER and SMD+HAMMER, respectively.

\begin{tabular}{|c|c|c|}
\hline & HAMMER & SMD+HAMMER \\
\hline \multirow{3}{*}{ Cluster 1 } & $P_{F W E-\text { corr }}=0.342$ & $P_{F W E-\text { corr }}=0.026$ \\
& $P_{F D R-\text { corr }}=0.057$ & $P_{F D R-\text { corr }}=0.003$ \\
& $T=12.91$ & $T=17.50$ \\
\hline \multirow{3}{*}{ Cluster 2 } & $P_{F W E-\text { corr }}=0.272$ & $P_{F W E-\text { corr }}=0.003$ \\
& $P_{F D R-\text { corr }}=0.057$ & $P_{F D R-\text { corr }}=0.003$ \\
& $T=13.26$ & $T=22.29$ \\
\hline
\end{tabular}

Table 1. Paired t-test results: p-values and t-values at the peaks of Cluster 1 in the superior temporal gyrus and Cluster 2 in the precentral gyrus

\subsection{Registration of real MR brain data}

\subsubsection{Measuring registration accuracy by manual landmarks}

We used 18 MR brain images from different subjects, each of which had 20 landmarks manually marked by experts. After registering these 18 images, we transformed all the manual landmarks onto the template domain and thus obtained 20 groups of corresponding points in the template domain, and each group consists of 18 points of the same landmark from 18 different subject images. For each group, we calculated the mean and std of the distances between all the 18 points and their average point; for ideal registration, these should be zero. These actual mean and std for each landmark group are shown in Fig.8. It can be seen that all the means are below $5 \mathrm{~mm}$, and similar registration accuracy was achieved for HAMMER and SMD+HAMMER ( $\mathrm{p}$-value for a paired t-test is 0.023). Notice that these results are comparable to the inter-rater errors in placing landmarks, i.e. the mean and std are $5.6 \mathrm{~mm}$ and $3.6 \mathrm{~mm}$,

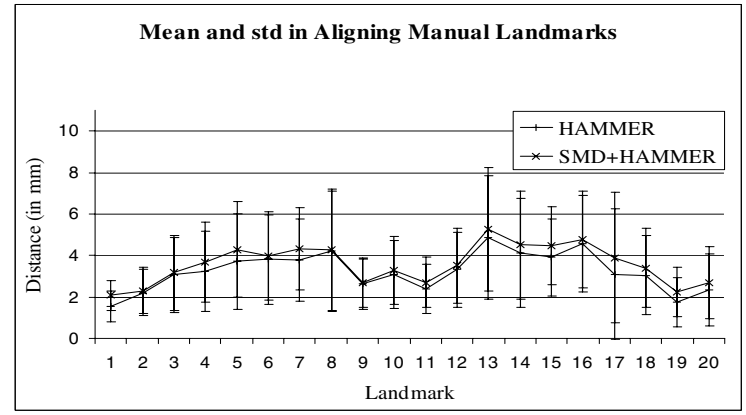

Figure 8. Comparison of the registration accuracy on manual landmarks.

respectively, for the same testing data (with voxel size of $1 \mathrm{~mm} \times 1 \mathrm{~mm} \times 2.2 \mathrm{~mm})$ [20].

\subsubsection{Registering serial MR brain images}

In this experiment, serial images of six different subjects are registered onto the template image by HAMMER and SMD+HAMMER, respectively, to compare the goodness of registration. Each subject has eight serial images captured from eight consecutive years rigidly aligned onto the template space. For accurately measuring subtle longitudinal changes, it is important to evaluate the temporal consistency of the registration results on the $Y$ serial images of each subject. Therefore, the Temporal Smoothness (TS) of the serial deformation fields, used to register the serial images of the same subject to the template, is measured as follows,

$$
T S(\mathbf{x})=\frac{1}{Y-2} \sum_{t=2}^{Y-1}\left|\mathbf{f}_{t}(\mathbf{x})-\frac{\mathbf{f}_{t+1}(\mathbf{x})+\mathbf{f}_{t-1}(\mathbf{x})}{2}\right| .
$$

A smaller TS value at $\mathrm{x}$ means the deformation fields along the corresponding voxels of $\mathbf{x}$ in the serial images are temporally smooth, while a large TS value means that deformation fields are not temporally smooth. Fig.9 gives an example of the TS map. Fig.9(a) and Fig.9(b) show the TS maps calculated from the serial deformation fields generated by using HAMMER and SMD+HAMMER, respectively. It can be seen that the TS map in Fig.9(b) has smaller values than that in Fig.9(a), e.g. smaller peaks and lower peak values are observed. We can also calculate the difference between the TS map of SMD+HAMMER and that of HAMMER, and overlay this difference of TS maps upon the template image, as shown in Fig.9(c). According to the color bar, we can observe that SMD+HAMMER can achieve temporally smoother results within red regions and yield similar results within yellow regions.

In fact, most values in the difference of TS maps are negative, indicating that SMD+HAMMER generates temporally smoother serial deformation fields for the serial images 


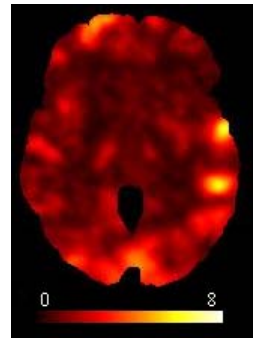

(a) HAMMER

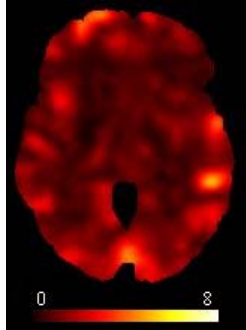

(b) SMD+HAMMER

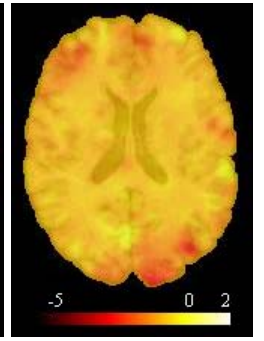

(c) Difference

Figure 9. Comparison of temporal smoothness of serial deformation fields. The difference of TS maps is defined as the subtraction of the TS map of SMD+HAMMER by the TS map of HAMMER.

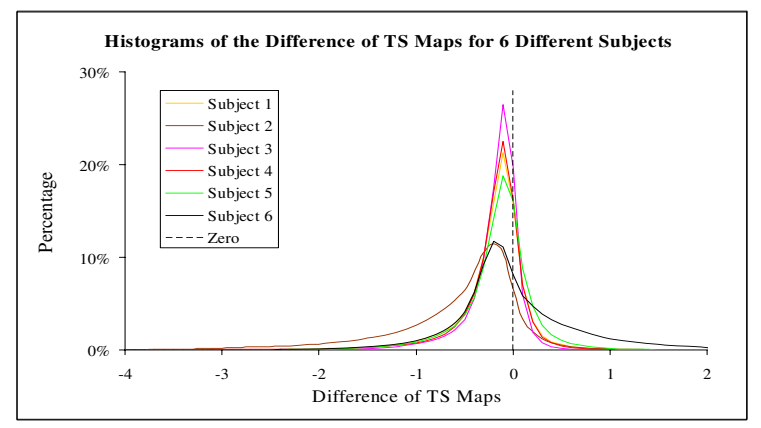

Figure 10. Histograms of the difference of TS maps for six different subjects.

than HAMMER. Alternatively, we can calculate the histogram of the difference of TS maps. Fig.10 shows six such histograms for six different subjects. It can be seen that for each subject, most values of the difference of the TS maps are below zero, thus most TS values of SMD+HAMMER are smaller than those of HAMMER.

It is important to note that the temporal consistency described above was not achieved by applying any temporal smoothness constraints or using any intra-individual statistics, and only the SMD trained from the inter-individual deformations was used. The temporally consistent registration results by SMD+HAMMER can also be visually observed using 3D rendering. Fig. 11 shows the template image and the registered serial images of the same subject in the template space, using a 3D rendering, for HAMMER and SMD+HAMMER, respectively. White contours are identically placed in each image, for facilitating the visual inspection. It can be seen that the shapes of the deformed gyri for the year 1, year 3 and year 5 of the HAMMER registration results (indicated by black arrows in Fig.11(a)) are quite different from those in the other years. In contrast, the shapes of the registered gyri by SMD+HAMMER are not only more temporally consistent, but also more similar with the template, as shown in Fig.11(b).

\section{Conclusion}

A statistically-constrained deformable registration algorithm is proposed to iteratively regularize the deformation field generated by a conventional registration algorithm using a statistical model, in which wavelet-based priors are used to capture the variability of deformations more accurately than the global PCA method. Experiments demonstrate that more robust registration results are obtained by the statistically-constrained registration, without decreasing the registration accuracy. The proposed method can potentially be incorporated into various registration algorithms to improve their robustness and stabilities by using statistically-based regularization.

\section{References}

[1] K. Boesen, J. H. S. Frey, J. Germann, J. Stern, D. L. Collins, A. C. Evans, and D. Rottenberg. Inter-rater reproducibility of $3 \mathrm{~d}$ cortical and subcortical landmark points. In 11th Annual Meeting of the Organization for Human Brain Mapping, Toronto, Canada, 2005. 2

[2] R. R. Coifman and M. V. Wickerhauser. Entropy-based algorithms for best basis selection. IEEE trans on Inf. Theory, 38:713-718, 1992. 1

[3] T. Cootes, A. Hill, C. Taylor, and J. Haslam. Use of active shape models for locating structures in medical images. Image and Vision Computing, 6(2):355-365, 1994. 1

[4] T. Cootes, C. Taylor, D. Cooper, and J. Graham. Active shape models - their training and application. Computer Vision and Image Understanding, 61(1):38-59, 1995. 1

[5] C. Davatzikos, A. Genc, D. Xu, and S. M. Resnick. Voxelbased morphometry using the ravens maps: Methods and validation using simulated longitudinal atrophy. NeuroImage, 14:1361-1369, 2001. 3, 4, 5

[6] C. Davatzikos, X. Tao, and D. G. Shen. Hierarchical active shape models using the wavelet transform. IEEE Transactions on Medical Imaging, 22:414-423, 2003. 1, 2

[7] J. Duncan and N. Ayache. Medical image analysis: Progress over two decades and the challenges ahead. IEEE Transactions on Pattern Analysis and Machine Intelligence, 22(1):85-106, 2000. 1

[8] A. F. Goldszal, C. Davatzikos, D. Pham, M. Yan, R. N. Bryan, and S. M. Resnick. An image processing protocol for the analysis of $\mathrm{mr}$ images from an elderly population. Journal of Computer Assisted Tomography, 22:827-837, 1998. 5

[9] M. Kamber, R. Shinghal, D. Collins, G. Francis, and A. Evans. Model-based 3D segmentation of multiple sclerosis lesions in magnetic resonance brain images. IEEE Transactions on Medical Imaging, 14:442-453, 1995. 1

[10] B. Karacali and C. Davatzikos. Estimating topology preserving and smooth displacement fields. IEEE Transactions on Medical Imaging, 23:868-880, 2004. 2

[11] S. Mallat. A wavelet tour of signal processing. Academic Press, 1998. 1 


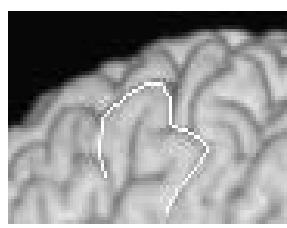

template image

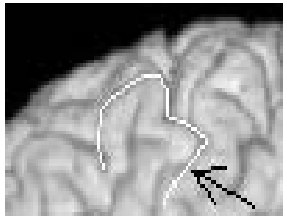

year 1

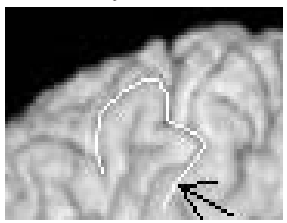

year 5

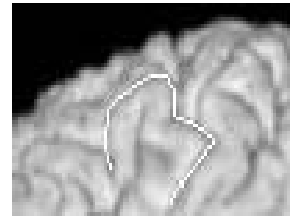

year 2

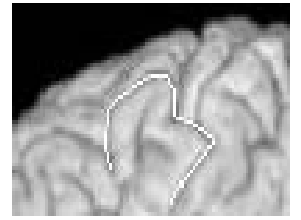

year 6

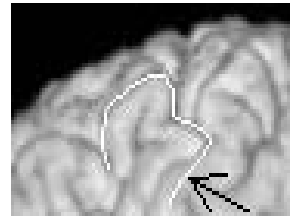

year 3

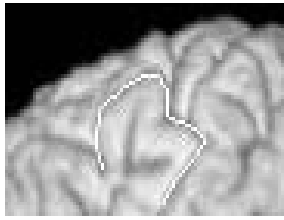

year 7

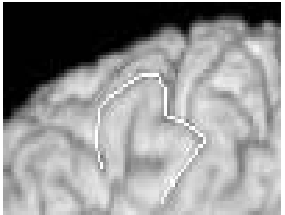

year 4

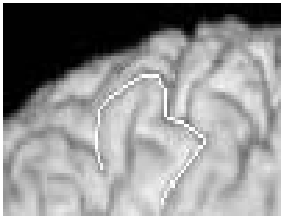

year 8

(a) Registered serial images by HAMMER

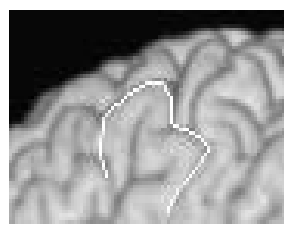

template image

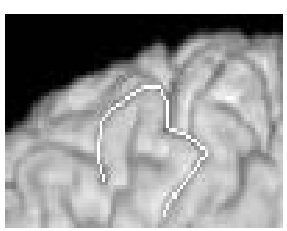

year 1

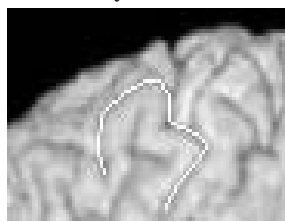

year 5

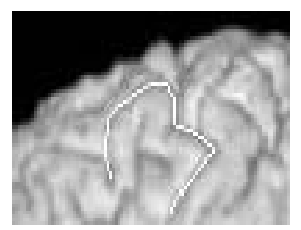

year 2

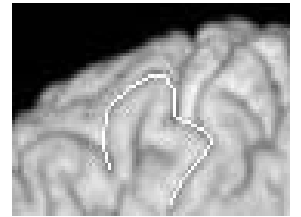

year 6

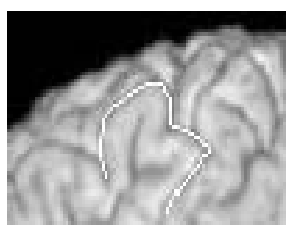

year 3

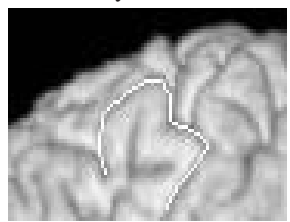

year 7

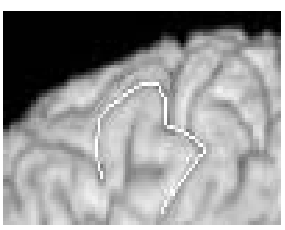

year 4

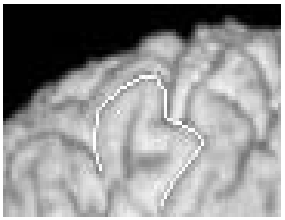

year 8

(b) Registered serial images by SMD+HAMMER

Figure 11. Warping consistency on eight-year serial images of the same subject.

[12] M. Miller, G. C. A. Banerjee, N. K. S. Joshi, U. Grenander, and L. Matejic. Statistical methods in computational anatomy. Statistical Methods in Medical Research, 6:267299, 1997. 1

[13] A. Mohamed and C. Davatzikos. Shape representation via best orthogonal basis selection. In Medical Image Computing and Computer Assisted Intervention(MICCAI), St. Malo, France, 2004. 1

[14] S. M. Resnick, A. Goldszal, C. Davatzikos, S. Golski, M. A. Kraut, E. J. Metter, R. N. Bryan, and A. B. Zonderman. Oneyear age changes in mri brain volumes in older adults. Cerebral Cortex, 10:464-472, 2000. 3, 5

[15] D. Rueckert, L. Sonoda, C. Hayes, C. Hill, M. Leach, and D.J.Hawkes. Nonrigid registration using free-form deformations: application to breast MR images. IEEE Transactions on Medical Imaging, 18(8):712-721, 1999. 1

[16] D. Shen and C. Davatzikos. HAMMER: Hierarchical attribute matching mechanism for elastic registration. IEEE Transactions on Medical Imaging, 21(11):1421-1439, 2002. 1,3

[17] A. Toga and P. Thompson. The role of image registration in brain mapping. Image and Vision Computing, 19(1):3-24, 2001. 1

[18] C. J. Twining, T. Cootes, S. Marsland, V. Petrovic, R. Schestowitz, and C. J. Taylor. A unified informationtheoretic approach to groupwise non-rigid registration and model building. In Information Processing in Medical Imaging 2005, Glenwood Springs, CO, 2005. 1

[19] P. Viola and W. Wells. Alignment by maximization of mutual information. In 5th International Conference on Computer Vision, ICCV'95, pages 16-23, 1995. 3

[20] Z. Xue, D. Shen, and C. Davatzikos. Determining correspondence in 3D MR brain images using attribute vectors as morphological signatures of voxels. IEEE Trans. on Medical Imaging, 23:1276-1291, 2004. 6

[21] Z. Xue, D. Shen, B. Karacali, , and C. Davatzikos. Statistical representation and simulation of high-dimensional deformations: Application to synthesizing brain deformations. In Medical Image Computing and Computer Assisted Intervention (MICCAI 2005), Palm Springs, California, USA, 2005. $1,2,3$ 